\title{
Volatilidad electoral y desafección política en las elecciones parlamentarias chilenas 1993-2009¹
}

\author{
Octavio Avendaño* \\ Pablo Sandoval**
}

\begin{abstract}
Resumen
La mayor parte de los estudios sobre el sistema de partidos y los procesos electorales realizados en Chile durante las últimas dos décadas han tendido a asumir que la estabilidad de los resultados de votación registrada entre 1989 y 2009 es el resultado de la permanencia en las preferencias electorales. Sin embargo, dicho supuesto se ve refutado por el incremento de la desafección generada desde la segunda mitad de los años noventa. Basándose en este antecedente, a lo largo del artículo se propone una explicación alternativa que permite entender la "paradoja de la estabilidad". Para ello, se lleva a cabo un análisis de encuestas electorales que incluyen preguntas retrospectivas, realizadas entre 1997 y 2009. Este análisis permite concluir que la estabilidad se ha mantenido en forma paralela al incremento en las tasas de voto no estable. Al mismo tiempo, se constata que los bajos índices de volatilidad electoral se sustentan en el carácter autocompensado del conjunto de cambios de movilidad de los votantes y de variaciones en la composición del electorado efectivo, lo que da cuenta de una diversificación en las formas de vinculación entre electorado chileno y sistema de representación política a lo largo del período.
\end{abstract}

Palabras clave: Chile - paradoja de la estabilidad - sistema de partidos - volatilidad electoral - voto no estable.

\begin{abstract}
Most of the studies on the party system and elections held in Chile over the last two decades have tended to assume that the stability of voting results recorded between 1989 and 2009 is the consequence of the permanence of electoral preferences. However, this assumption is refuted by the increasing disaffection generated from the second half of the nineties. Based on this background, throughout the article an alternative explanation that allows understanding the "stability paradox" is proposed. For this purpose, an analysis of electoral surveys made between 1997 and 2009 -which include retrospective questions-is conducted. This analysis leads to the conclusion that stability is maintained in parallel to the increase in non-stable voting rates. At the same time, it appears that low levels of electoral volatility are based on the self-compensated character of the set of voters' mobility changes and variations in the composition of the effective electorate, which give account for a diversification in the forms of linkage between Chilean electorate and the political representation system over the period.
\end{abstract}

Keywords: Chile - paradox stability - party systems - electoral volatility - non-stable vote.

$1 \quad$ Este artículo es resultado de una investigación financiada por la Vicerrectoría de Investigación y Desarrollo (VID) de la Universidad de Chile, la que llevó por título: "Adhesión e identificación partidaria en Chile. Estudio acerca de la volatilidad y la movilidad electoral en el período 1989 a 2009" (Proyecto SOC 10/11-2). Se agradecen los comentarios de Gonzalo Robledo, Mauricio Morales y Mariano Torcal. También agradecemos al profesor Carlos Huneeus por permitirnos acceder a las encuestas del Centro de Estudios de la Realidad Contemporánea (CERC).

* Doctor en Ciencia Política, Universidad de Florencia, Italia. Académico del Departamento de Sociología de la Universidad de Chile y del Departamento de Ciencia Política y Relaciones Internacionales de la Universidad Alberto Hurtado. Investigador del Proyecto VID SOC 10/11-2. Correos electrónicos: oavendanop@u.uchile. cl, oavendan@uahurtado.cl

** Sociólogo, Universidad de Chile. Investigador del Proyecto VID SOC 10/11-2. Correo electrónico: psandovalv@ live.cl 


\section{INTRODUCCIÓN}

En el plano electoral, Chile se ha caracterizado durante las últimas décadas por la estabilidad de los resultados de votación obtenidos por las principales coaliciones presentes desde el retorno de la democracia. Esta situación ha sido destacada por diversos estudios que han descrito al sistema chileno como uno de los que presentan los índices más bajos de volatilidad electoral en el contexto latinoamericano (Alcántara, 2004; Jones, 2005; Mainwaring y Torcal, 2005; Mainwaring y Zoco, 2007).

A partir de esta constatación se ha tendido a asumir que los bajos índices de volatilidad se originan en la preeminencia de un comportamiento de voto estable a nivel del electorado. En otras palabras, los resultados de votación mantendrían estabilidad debido a que la mayor parte del electorado votaría por candidatos de la misma coalición política entre una elección y otra. Según perspectivas como la de Garretón (2000), lo anterior daría cuenta de la vigencia de un marco de fidelidades electorales políticamente estructuradas a nivel de la vinculación entre votantes y agentes de representación política.

No obstante, este diagnóstico contrasta con la imagen de un electorado crecientemente distanciado de bloques y partidos políticos, como lo muestran evidencias de numerosos estudios realizados desde mediados de la década de los noventa. De acuerdo con estos antecedentes, durante las últimas décadas los niveles de filiación e identificación política han disminuido drásticamente, mientras que los de desafección y desconfianza hacia bloques y partidos se han incrementado (Morales, 2010). Asimismo, se ha señalado que los índices de desafección presentes en el sistema chileno resaltan por sobre los existentes en otras democracias latinoamericanas (Luna y Altman, 2011).

¿Cómo se explica la mantención de la estabilidad electoral en un contexto de progresivo incremento de la desafección política? Una explicación proporcionada por la literatura especializada utiliza el concepto de "democracia de baja intensidad" para aludir a la mantención de las fidelidades electorales pese a la caída de la identificación política (Luna, 2008: 89). Otros trabajos han acuñado el término "paradoja de la estabilidad" para referir al carácter irresuelto de la problemática (Inap-Segpres, 2008; Joignant y López, 2005; López, 2008).

Nuestra intención con este trabajo consiste en explorar una hipótesis alternativa, resaltando las aristas sociológicas del problema, esta es: que la estabilidad electoral puede haberse mantenido en Chile en paralelo a la erosión de las fidelidades electorales, siendo esto último la expresión en el plano electoral del incremento de la desafección política. Esta proposición se fundamenta en trabajos como los de Crewe y Denver (1985) y Bartolini y Mair (1990), quienes señalaron que la estabilidad de los resultados de votación puede convivir en un sistema electoral con considerables tasas de voto no estable. Desde ambas contribuciones se desprende la existencia de dos tipos de votantes no estables:

1. Los primeros son aquellos que modifican su comportamiento de votación en una elección 2, favoreciendo a una opción política diferente a la escogida en la elección 1 , a los cuales Ilamamos en este trabajo "votantes móviles". 
2. El segundo tipo corresponde al elector que emite un voto válido en solo una de dos elecciones consecutivas analizadas (ya sea en la primera o en la segunda) absteniéndose, o no participando del proceso electoral en la otra.

Una importante presencia de ambos perfiles de votantes en la composición del electorado puede generar considerables índices de volatilidad electoral, a menos que el resultante de este conjunto de cambios se vea autocompensado a nivel agregado. Así, si por cada votante que transfiere su voto de A hacia B existe otro votante que recorra el camino inverso, la movilidad electoral no generará volatilidad ${ }^{2}$. Lo mismo ocurre con el segundo perfil de electores: si una coalición pierde un considerable número de votos hacia el abstencionismo, pero en compensación de ello logra captar el equivalente número sobre votantes ausentes en la elección anterior, el cambio en su votación no habrá de ser significativo y, por ende, la volatilidad del sistema no variará considerablemente. De allí, como ha señalado Roblizo (2001), que bajos e inclusive nulos niveles de volatilidad puedan esconder un alto, pero autocompensado, nivel de cambio en el comportamiento de votación del electorado.

¿Puede haber sido este el caso de la competencia electoral desarrollada en Chile en el periodo comprendido desde la restauración de la democracia hasta las últimas elecciones parlamentarias y presidenciales de 2009? Como hipótesis, esta proposición encuentra asidero en una serie de estudios que señalan que el porcentaje de votantes que no mantiene un comportamiento de voto estable resultaría sustancialmente más alto que lo expresado en los índices de volatilidad electoral (Huneeus, 2002; Joignant y López 2005; López, 2008; López y Baeza, 2011). De acuerdo con ello, la confluencia entre desafección política y estabilidad electoral se explicaría en virtud del carácter autocompensado del conjunto de cambios de movilidad electoral y de composición del electorado efectivo, los cuales vendrían incrementándose en Chile en paralelo al deterioro en los niveles de identificación y confianza con bloques y partidos. De ser efectivo, esto último indicará, como ha señalado Luna (2008), que la vinculación entre electorado y sistema de representación no se estructura en función de la preeminencia de un componente político-programático, sino más bien que ha experimentado una diversificación en el transcurso de las últimas décadas, adquiriendo en algunos casos, inclusive, un carácter de corto plazo.

La investigación cuyos resultados se presentan en este artículo consistió en evaluar la composición del electorado, distinguiendo entre votantes estables y no estables, para cada par de elecciones de cámara baja (consecutivas) realizadas entre 1993 y 2009. Al comparar estos datos con las cifras de volatilidad electoral se buscó, en primer lugar, conocer el sustento de la estabilidad de los resultados electorales durante el período, y luego, problematizar el tipo de vinculación entre electorado y sistema de representación. Para cumplir con este objetivo, el presente trabajo se estructura en cuatro grandes apartados. En el primero se analizan las principales interpretaciones y antecedentes acerca de la confluencia entre estabilidad electoral y desafección política en la democracia chilena. En el segundo apartado se alude a los criterios metodológicos utilizados en el estudio. En la tercera sección se presentan los

2 O la generará en términos moderados en caso de que la transferencia inversa de electores no sea de suma cero. 
principales resultados alcanzados con la metodología adoptada. Finalmente, se desarrollan las conclusiones más relevantes extraídas a partir del análisis de la información obtenida.

\section{DISCUSIONES EN TORNO A LA ESTABILIDAD, LA DESAFECCIÓN Y EL VOTO NO ESTABLE}

\subsection{El diagnóstico de la estabilidad electoral}

Por volatilidad electoral es posible entender el nivel de cambio en las votaciones obtenidas por los agentes de representación política (bloques, partidos) instituidos en un sistema electoral en dos elecciones consecutivas. Usualmente, la volatilidad es medida a través del Índice de Pedersen $(1979)^{3}$, el cual constituye un importante indicador utilizado para el estudio del grado de institucionalización y arraigo social de los sistemas de partidos en democracias de diferentes regiones del mundo.

A partir de la década de los noventa, en Chile diversas mediciones han utilizado el Índice de Pedersen para el estudio de la dinámica del sistema electoral. Al basarse en esta medida común, los resultados de dichos estudios resultan altamente coincidentes. Mainwaring y Scully (1996) cifran en 15,8\% la volatilidad electoral promedio entre las elecciones de cámara baja a nivel de partidos en el periodo 1973-19934. A su vez, Alcántara (2004) estima un promedio de 14,1\% entre 1989 y 2001, en el mismo tipo de elecciones, también a nivel de partidos. Mediciones posteriores entregan cifras más bajas. De acuerdo con Joignant (2010) la volatilidad promedio entre 1993 y 2005 es de 7,8\%, misma cifra que la estimada por Luna (2008) para el intervalo 2001-2005. Otras estimaciones han dado cuenta de la existencia de niveles de volatilidad aún más bajos, al considerar esta vez las variaciones de los resultados electorales a nivel de las principales coaliciones políticas presentes en el período. Al respecto, trabajos como los de Jones (2005) y Joignant (2010) constatan una volatilidad interbloques en elecciones de cámara baja entre los años 1989 y 2005 cercana al 6\%.

En vista a los valores observados en el caso chileno, gran parte de los analistas especializados en la temática han tendido a asumir que la continuidad de los resultados de votación responde a que la mayor parte de los electores votaría por la misma opción política entre una elección y otra. Lo anterior se relaciona con la interpretación instalada en la literatura especializada acerca de las causas que dan lugar a sistemas electoralmente estables. Tradicionalmente se ha tendido a asumir que dichos sistemas presentan bajos índices de volatilidad producto de la existencia de organizaciones de representación política con altos índices de raigambre y adhesión social (Mainwaring y Scully, 1996). En su versión más simplificada, esta perspectiva

3 El Índice de Pedersen se calcula sumando las diferencias de votación, en valores absolutos, obtenidas por los partidos (o bloques) instituidos en un sistema electoral, en dos elecciones sucesivas, y dividiendo luego dicha sumatoria por 2. Su rango oscila entre $0 \%$ y $100 \%$ (donde $0 \%$ implica nula volatilidad y $100 \%$ la máxima). En términos algebraicos: $V=\frac{1}{2} \sum_{i=1}^{n} P_{i} \bar{V}$, donde la variación de votación que experimenta un partido $P_{i}$ se representa por: $P_{i} \bar{V}=\left|P_{i}(t+1)-P_{i}(t)\right|, i=1, \ldots . n$.

4 Los autores califican el anterior resultado como "moderadamente estable" considerando el rango regional dado por el $9,1 \%$ de Uruguay y el $54,4 \%$ de Perú para períodos similares. 
propone una equivalencia entre el valor asumido por el Índice de Pedersen y el porcentaje de votantes que modifican su comportamiento de votación entre dos elecciones sucesivas. De este modo, una volatilidad de $6 \%$ indicaría que solo un $6 \%$ de los votantes cambiaría su comportamiento de votación en el intervalo electoral correspondiente.

Coincidiendo en este tipo de interpretación acerca de los niveles de volatilidad electoral observados en el caso chileno, la literatura especializada difiere luego en torno al origen de estos alineamientos. Al respecto, es posible identificar tres tipos de proposiciones que pretenden explicar esta situación. La primera considera que la estabilidad electoral se explica por la reemergencia en el periodo post-autoritario de los alineamientos originados por los clivajes sociales ${ }^{5}$ que estructuraron el sistema político con anterioridad al quiebre democrático. De acuerdo con este enfoque, el sistema de partidos chilenos se conformó a partir de un conjunto de "fisuras generativas", como fueron el conflicto religioso de finales del siglo XIX y los de clases sociales de carácter urbano y rural durante el siglo XX, los cuales, producto de su hibridación, habrían dado origen a una división tripartita entre izquierda, centro y derecha, a la vez que a un alto grado de fidelidades electorales para con las opciones partidistas representativas de dicha división (Scully, 1996: 83). Trabajos como los de Scully y Valenzuela (1993), Scully (1996), Mainwaring y Scully (1996) y Valenzuela $(1995,1999)$ coinciden en señalar que el escenario presente con el retorno de la democracia significó la reemergencia de dichos alineamientos, con independencia en el cambio de los rótulos de los partidos y de su agrupamiento en coaliciones políticas.

En oposición a este planteamiento, una segunda proposición relaciona los bajos índices de volatilidad con la estructuración de un nuevo clivaje, de carácter eminentemente político ${ }^{6}$, basado en la oposición entre autoritarismo y democracia. Producto del advenimiento de este eje de conflicto, los antiguos alineamientos partidarios serían reemplazados por un nuevo marco de fidelidades electorales, ahora en torno a las principales coaliciones constituidas con el retorno del sistema democrático (Huneeus, 2003; Tironi y Agüero, 1999; Tironi et al., 2001). Esta proposición cobró fuerza a partir de diversos análisis que constataron la preponderancia de las identidades políticas asociadas a este eje de conflicto, versus el decaimiento de los factores tradicionales (clase social y religión), como principal determinante del comportamiento de voto durante el periodo7 (Angell y Reig, 2007; Gutiérrez y López, 2007; Huneeus, 2003; Joignant, 2010; Joignant y López, 2005; López, 2004, 2008; Ortega, 2003).

En paralelo a las anteriores explicaciones, una tercera proposición atribuye los bajos índices de volatilidad electoral a la vigencia del sistema electoral binominal, mecanismo

5 Desde la perspectiva de la teoría de los clivajes, elaborada inicialmente por Lipset y Rokkan (1967), la estabilidad electoral y la del sistema de partidos dependen de si una fractura (o fisura) se mantiene congelada (frozen cleavage) a lo largo de un período más o menos prolongado. Lipset y Rokkan identificaron una serie de fracturas sociales que estructuraron la dinámica del sistema de partidos europeo durante los siglos XIX y XX, que los partidos intentaron siempre asumir y representar, como el religioso, el demográfico o el de clases.

En contraposición al carácter sociológico de los anteriores.

Al respecto, Gutiérrez y López señalan: "la estabilidad que a nivel agregado se ha podido observar desde el retorno del régimen democrático, tiene, en el nivel de los votantes, explicación en identidades y actitudes de tipo político" (Gutiérrez y López, 2007: 184). 
constituido con el fin de delimitar las posibilidades de fragmentación del escenario político, al incentivar la competencia en grandes coaliciones y desfavorecer las candidaturas no adheridas a aquellos (Gamboa, 2006; Ortega, 2003). De este modo, la continuidad de los resultados de votación se explicaría también por la delimitación de la oferta políticoprogramática inducida por el binominalismo, al establecerse como un mecanismo que obliga a los electores a escoger entre una oferta electoral reducida.

Con todo, el análisis de la estabilidad electoral presente en Chile desde el retorno de la democracia ha recurrido a una complementariedad entre proposiciones que apelan a la vigencia de determinado tipo de clivaje y a la incidencia del sistema electoral binominal para explicar la continuidad de los resultados de votación. El denominador común a todas estas explicaciones consiste en concebir a la estabilidad electoral como el resultado de un comportamiento de voto estable a nivel del electorado.

\subsection{Desafección política y estabilidad electoral}

Contrario a la idea de un afianzamiento de las preferencias de votación, asumida por las diferentes explicaciones sobre los bajos índices de volatilidad presentes en Chile, existe un conjunto de evidencias que dan cuenta de una situación de progresiva desafección, malestar y desconfianza por parte del electorado y la ciudadanía en general hacia los agentes de representación política instituidos en el periodo (Angell, 2003; Huneeus, 1999; Luna, 2008; Luna y Altman, 2011; Morales, 2010; Ortega, 2003; Siavelis, 1999). Esta tendencia se observa desde mediados de los noventa, y según Siavelis se ha expresado, entre otros aspectos, en un decaimiento en la identificación y confianza con partidos y coaliciones políticas, en el advenimiento de un electorado menos ideológico, y en crecientes tasas de abstencionismo y de "voto protesta" (Siavelis, 1999: 245). A nivel comparativo se ha señalado también que los niveles de desafección política presentes en Chile destacan por sobre los existentes en otros países de la región (Luna y Altman, 2011; Luna, 2008; Luna et al., 2010). Según estos antecedentes ${ }^{8}$, el electorado chileno se caracteriza por una mayor lejanía con bloques y partidos, por bajos índices de militancia partidaria y de participación ciudadana en campañas políticas, e incluso, por una menor adhesión a la democracia como régimen político.

Considerando estas evidencias, trabajos como los de Luna (2008), López (2008) y Morales (2010) han destacado el carácter contradictorio del incremento de la desafección política en un contexto donde los resultados electorales siguen manteniendo estabilidad. De acuerdo con Luna, "la presencia de un sistema de partidos débil en cuanto a su relación con la sociedad civil y sumamente fuerte en cuanto a reproducir lealtades electorales (tal como señala el índice de volatilidad), es aparentemente paradójica. En otras palabras, no parece usual que un sistema de partidos estable e institucionalizado a nivel electoral conviva con una ciudadanía crecientemente alejada de los partidos y sin simpatías partidarias cristalizadas" (Luna, 2008: 88).

8 Obtenidos a partir de los resultados de las encuestas LAPOP realizadas entre los años 2006 y 2010. 
Los análisis del mismo Luna esbozan una serie de explicaciones para este problema. Para ello propone centrar la discusión entre los vínculos entre partidos y sociedad, los que, de acuerdo con Montes, Mainwaring y Ortega (2000) no habrían presentado previo al quiebre democrático los niveles de estructuración programática supuestos por la perspectiva de los clivajes sociales ${ }^{9}$. El autor sugiere para el período una situación de progresiva diversificación en el tipo de vinculación, según la cual sería posible observar un tránsito desde la preeminencia de vínculos colectivos programáticos, partidarios y no partidarios, a fines de los ochenta, hacia una "nueva configuración" que combina tanto vínculos colectivos como de carácter individual, partidarios y no partidarios, como así también programáticos y no programáticos (Luna, 2008: 112).

Si bien esta última proposición permite comprender la erosión de los vínculos programáticos desde una perspectiva más comprehensiva que la dicotomía vigencia/no vigencia del clivaje político, la "paradoja de la estabilidad" es abordada solo a nivel descriptivo, sin lograr una explicación más completa. La pregunta parece ser entonces: si se produce una erosión de los vínculos programáticos, que se expresa entre otros aspectos en una progresiva desafección política, ¿por qué los resultados electorales siguen manteniendo estabilidad? En torno a este asunto, Luna y Altman (2011) agregan un nuevo elemento a la discusión, planteando una crítica al uso dado al concepto de volatilidad electoral para evaluar el grado de estabilidad de las preferencias de votación. De acuerdo con los autores, la volatilidad no expresa necesariamente la real magnitud de transferencias electorales individuales, pudiendo aquellas asumir valores sustancialmente mayores a los reflejados por el Índice de Pedersen. De acuerdo con ello, el estatus de "paradoja" definido por la confluencia entre estabilidad electoral y desafección política respondería a una conceptualización errónea de la volatilidad como indicador de asentamiento de lealtades partidarias (Luna y Altman, 2011: 22). El problema asociado a la "paradoja de la estabilidad" radicaría entonces en atribuir estabilidad al comportamiento de voto del electorado a partir de la constatación de la existencia de bajos índices de volatilidad electoral. Por el contrario, de acuerdo con lo señalado por Luna y Altman, y anteriormente por Crewe y Denver (1985) y Bartolini y Mair (1990), la estabilidad de los resultados de votación puede llegar a convivir con un importante nivel de cambio en el comportamiento de votación del electorado.

\subsection{El aumento del voto no estable}

El concepto de voto no estable alude directamente al comportamiento que manifiestan los electores frente a las opciones políticas existentes entre una elección y otra. En términos simples, el voto no estable implica no sufragar por la misma opción política en dos o más elecciones consecutivas. Este fenómeno fue abordado en Chile por Martínez y Palacios

$9 \quad$ Este trabajo indica que, aun presentando considerables grados de estructuración política, la relación entre partidos y sociedad en Chile previo al quiebre democrático vio convivir vínculos de carácter político-programático con otros de carácter clientelar. De allí que resulte parcialmente erróneo suponer la reemergencia de un vínculo políticamente estructurado en la democracia post 1989, ya sea en la forma de los determinantes tradicionales, o bajo el contenido del clivaje político. 
(1991) tras las primeras elecciones parlamentarias celebradas en 1989. En el marco de dicho estudio se pudo advertir la presencia de un sector minoritario del electorado que manifestaba retraimiento y apatía respecto de los procesos electorales, segmento en el que se correspondían marginalidad social y política, expresada esta última en términos de desconocimiento y desinterés ante la lógica del sistema democrático. En adelante, resultados de otros estudios señalaron que el comportamiento de voto no estable se expandió durante las últimas décadas más allá del nicho de marginalidad social identificado por Martínez y Palacios. Así lo indican análisis realizados por Tironi, Agüero y Valenzuela, para quienes el "voto migrante" adquiere relevancia desde mediados de la década de los noventa e incluso un perfil social relativamente definido $(2001: 86)^{10}$.

Investigaciones posteriores ratificaron esta tendencia. De acuerdo con Joignant y López (2005) la elección parlamentaria de 2001 marca una discontinuidad respecto al alto grado de estabilidad en el comportamiento electoral presente con anterioridad a dicho evento ${ }^{11}$. En torno a esta misma data, evidencias aportadas Huneeus (2002) reflejan un alto nivel de cambio en el comportamiento electoral de votantes de todos los partidos del espectro político, lo que reflejaría un desencanto con el desempeño de estas organizaciones y con el funcionamiento del sistema democrático en su conjunto ${ }^{12}$. En relación con este asunto, Huneeus propone una importante diferenciación entre "desencantados activos" y "desencantados pasivos", correspondiendo los primeros a aquellos que cambian sus preferencias de votación hacia otros partidos, mientras que los segundos transformarían su desencanto en abstención.

Antecedentes de carácter más reciente indican que cerca de un 35\% del electorado no mantendría estabilidad en su comportamiento de votación, versus un $65 \%$ que reconocería votar por candidatos del mismo partido (Inap-Segpres, 2008; López, 2008). No obstante, la lectura de este tipo de datos ha tendido a enfatizar la permanencia de un comportamiento estable a nivel del electorado, debido a que el porcentaje de votantes que reconoce votar por el mismo partido es mayor al de quienes reconocen una filiación político-partidista. Se omite, de esta forma, una explicación que intente relacionar este porcentaje de voto no estable con la continuidad de los resultados electorales presentes a lo largo del período.

Llegado a este punto, resurge la interrogante dada por el incremento del voto no estable y la mantención de la estabilidad de los resultados electorales. Ciertamente, a la luz de las evidencias anteriormente presentadas, el modelo de la estabilidad del votante como causa de la estabilidad electoral agregada queda en entredicho, o al menos a condición de que consideremos que la existencia de un $65 \%$ de voto estable no constituye una primacía capaz

10 Correspondiente a mujeres de segmentos de ingreso medio-bajos, con bajos niveles de calificación.

11 Cerca de un millón de electores habrían modificado su comportamiento de voto - de un total de algo más de seis millones de votos válidamente emitidos- principalmente a favor del bloque Alianza por Chile. No obstante, diagnosticar un realineamiento de las preferencias electorales a partir del panorama de 2001 hubiese resultado precipitado, tal como indica la importante declinación de la votación del bloque derechista en la elección Municipal de 2004, en comparación con la obtenida en la elección parlamentaria precedente (Joignant y López, 2005: 69-70).

12 De acuerdo con el Informe de Desarrollo Humano 2002, esta imagen coincide con la del "ciudadano desvinculado", que se desafecta de la política y que no espera nada de la democracia. De acuerdo con este informe, el "ciudadano desvinculado" representaría a un 27\% de la población (PNUD, 2002: 273). 
de traducirse por sí sola en la estabilidad de los resultados agregados. Con ello resurge también el término "paradoja de la estabilidad" para hacer referencia a esta situación.

En torno a este asunto, nuestra proposición apunta a que el comportamiento de voto no estable se fue incrementando durante las últimas décadas, pero que por presentar flujos de modificación de signo inverso, la tendencia se ha visto compensada en el valor de volatilidad de nivel agregado. De ser efectivo, esto podría verificarse en tres tipos de tendencias: a) la mantención de bajos índices de volatilidad electoral; b) la disminución en el porcentaje de votantes que mantienen un comportamiento de voto estable; y c) en la existencia de un carácter autocompensado entre el conjunto de cambios de movilidad electoral y de composición del electorado efectivo.

\section{CRITERIOS METODOLÓGICOS PARA ANALIZAR LA VOLATILIDAD Y EL VOTO NO ESTABLE}

La metodología buscó comparar el comportamiento de voto del electorado entre cada par de elecciones parlamentarias celebradas desde 1989. Existe una serie de dificultades metodológicas asociadas a este propósito. Desde la literatura internacional se plantea que las condiciones óptimas para la realización de un estudio como el propuesto dependen de la disposición de un panel de votantes sobre el cual realizar contrastes de carácter longitudinal (Powell y Tucker, 2009; Roblizo, 2001; Shair-Rossenfield, 2008). No obstante, como se ha señalado en estudios previos, en el medio nacional se carece de este tipo de antecedentes (Inap-Segpres, 2008). Frente a ello, una opción consiste en trabajar con encuestas electorales que incluyan preguntas retrospectivas. En este estudio se utilizó este tipo de datos, provenientes de las encuestas del Centro de Estudios de la Realidad Contemporánea (CERC) previo a cada elección parlamentaria realizada desde 1997. Dichos sondeos, de cobertura nacional, contienen preguntas sobre intención de voto por partido político para la elección de cámara baja correspondiente a dicho año, y otra pregunta en la que se le solicita al encuestado recordar el partido por el que votó en la elección parlamentaria anterior. Este tipo de datos no fueron recogidos por CERC para el período 1989-1993, lo cual nos llevó a circunscribir el análisis al intervalo de votaciones realizadas entre 1993 y 2009 y a nivel de elecciones de cámara baja.

\subsection{Tipología de votantes}

Para realizar el análisis se procedió a cruzar el comportamiento de voto declarado para cada par de elecciones comprendidas en el período señalado ${ }^{13}$. A partir de este procedimiento

13 Este cruce se basa en las posibilidades de comportamiento de votación del electorado en la segunda de dos elecciones consecutivas del mismo tipo (elección 2). En esta votación dicho electorado puede: i) votar por una opción política; o bien, ii) no votar por una opción política (voto nulo, blanco, o no concurriendo a votar). Ello abarca la totalidad de posibilidades de comportamiento del conjunto de electores que formen parte del padrón en una elección 2. En contraste, este mismo electorado puede haber presentado las siguientes opciones de comportamiento en la elección precedente: i) haber votado por una opción política; ii) no haber emitido un voto válido por una opción política (en los mismos términos antes descritos); o bien, iii) no haber formado 
fue posible discernir una tipología de votantes según mantuvieran estos concordancia o discordancia en su situación para efectos del recuento electoral en ambos comicios. Para ejemplificar esta situación (Tabla 1) supongamos un escenario donde dos opciones políticas, A y B, compiten en dos elecciones consecutivas. Se trata de la composición del electorado en la elección 2.

TABLA 1

Tipología de votantes

\begin{tabular}{|c|c|c|c|c|c|}
\hline \multicolumn{6}{|c|}{ ELECCIÓN 1} \\
\hline \multirow{4}{*}{ ELECCIÓN 2} & & A & B & NINGUNO & NO INSCRITO \\
\hline & A & I & II & III & III \\
\hline & B & II & I & III & III \\
\hline & Ninguno & III & III & IV & V \\
\hline
\end{tabular}

Los votantes tipo I son aquellos que votan por la misma opción política en ambas elecciones (votante estable). Los electores del tipo II, en cambio, modifican su preferencia de votación, de modo que su comportamiento de voto en la elección 2 favorece a una opción política diferente a la escogida en la elección precedente (votante móvil). Los electores tipo III son aquellos que votan en solo una de las dos elecciones consideradas, debido a que no forman parte del padrón en la elección 1, o a que no votan por una opción política en alguna de las dos elecciones (no concurren a votar o no emiten un voto válido) ${ }^{14}$. Por su parte, los votantes tipo IV no votan o no emiten un voto válido en ninguna de las dos elecciones, pese a que forman parte del padrón en ambos comicios (votante desvinculado). Finalmente, los electores tipo $\mathrm{V}$ son aquellos que no votan en ninguna de las elecciones, puesto que no formaban parte del padrón en la elección 1 y luego no emiten un voto válido en la elección 2.

El conteo sobre el total de tabla de los casos pertenecientes a cada perfil de votantes origina indicadores por medio de los cuales evaluar las diferentes hipótesis asociadas al problema estudiado. El modelo de la estabilidad del votante se verificaría en caso de que las casillas correspondientes al votante tipo I se acerquen a concentrar el $100 \%$ de los casos del total de la tabla, o bien, al complemento de 100\% del nivel de volatilidad electoral para el intervalo electoral correspondiente. En la medida en que la distribución de la tabla

parte del padrón electoral en la elección 1 (lo que en términos de la institucionalidad electoral correspondiente al periodo estudiado equivale a no haber estado inscrito en los registros electorales).

14 Nótese que dentro de la clasificación de votantes tipo III se encuentra ausente el perfil de aquellos electores que abandonan el padrón en la elección 2 debido a fallecimientos o abandonos legales. Esta es una limitante inherente a una estrategia de análisis con preguntas retrospectivas. En el marco de este trabajo supondremos que la ausencia de datos de este subsegmento no altera sustancialmente la composición del perfil de votantes derivado del cruce de información correspondiente a ambos comicios. 
se aleje de presentar dicha estructura, la hipótesis de la estabilidad del votante debiese ser descartada. En cambio, una presencia importante de votantes tipo II y/o III (voto no estable) indicará que existe un considerable nivel de cambio en el comportamiento de los votantes. Al respecto, un análisis previo realizado por los autores del presente trabajo en relación con la estructura interna del Índice de Pedersen (Avendaño y Sandoval, 2011), permitió concluir que si en un sistema electoral confluyen estabilidad electoral y una alta presencia de votantes no estables, los bajos índices de volatilidad responderán, necesariamente, al carácter autocompensado de los flujos de modificación de los votantes tipo II y/o III. Sobre la base de esto último, la estrategia de interpretación de los datos opera bajo la siguiente lógica: si la presencia de votantes estables no es todo lo predominante que cabría esperar, y si los resultados electorales siguen siendo estables, ello indicará que la estabilidad se produce, efectivamente, por la existencia de dicho efecto autocompensatorio.

Un ejemplo gráfico permitirá representar de mejor forma esta situación de "estabilidad dinámica". El siguiente esquema muestra las posibles "direcciones" del voto no estable para la competencia entre dos bloques A y B. Los flujos horizontales representan los cambios de movilidad electoral (tipo II), mientras que los verticales corresponden a los cambios de votantes tipo III.

FIGURA 1

Direcciones del voto no estable

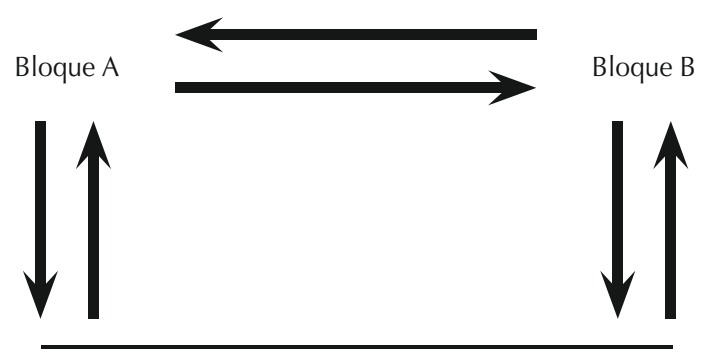

Nulo - Blanco - No concurrente / No inscrito

\subsection{Ajuste de datos}

El análisis se realizó a nivel de bloques políticos, lo que implicó agrupar los partidos correspondientes a cada conglomerado, al ser la pregunta original contenida en las encuestas CERC. Las respuestas fueron codificadas en cuatro opciones, correspondientes a bloques: i) Concertación, ii) Alianza, iii) Izquierda y iv) $\operatorname{Otros}^{15}$. Se excluyó del análisis aquellos casos

15 Se decidió optar por este nivel de análisis debido a que se trabaja con información de elecciones de cámara baja, donde, a partir de la incidencia del sistema electoral binominal, los cambios de votación entre partidos del mismo bloque resultan menos expresivos de la problemática estudiada que los cambios a nivel de bloques. 
que presentaron problemas de respuesta ${ }^{16}$, trabajando en definitiva con cerca del $75 \%$ de cada muestra ${ }^{17}$. Por su parte, los resultados fueron ponderados según los resultados reales de las elecciones 2, para lo cual se consideraron los resultados a nivel de la totalidad del electorado, es decir, contemplando votos válidamente emitidos, votos nulos, blancos y el número de electores que no acudieron a sufragar (agrupando a estos tres últimos bajo la categoría “Ninguno"). Considerando esta limitante (ponderación), el análisis de los indicadores dados por el recuento sobre el total de tabla de los diferentes perfiles de votantes se realiza en búsqueda de tendencias generales -las que se supone se mantienen en las muestras pese a los ajustes realizados sobre ellas- $y$ no de porcentaje o cifras exactas ${ }^{18}$.

Finalmente, se calculó la volatilidad electoral interbloques considerando las 4 opciones políticas antes señaladas, para cada par de elecciones de cámara baja realizadas desde 1993. Para ello se utilizó la formulación del Índice de Pedersen a nivel de conglomerados. El análisis que sigue consiste en contrastar dichos índices de volatilidad con la composición del electorado de acuerdo con la tipología antes presentada, en busca de evaluar el sustento de la estabilidad electoral agregada a nivel del comportamiento de los votantes y el tipo de vinculación existente entre votantes y sistema de representación.

\section{VOLATILIDAD Y COMPORTAMIENTO ELECTORAL 1993-2009}

\subsection{Estabilidad y voto no estable 1993-2009}

Al calcular la volatilidad electoral interbloques para cada par de elecciones de cámara baja consecutivas realizadas desde 1993 constatamos la estabilidad que mantienen los resultados de votación, aunque con ciertas fluctuaciones. Entre las elecciones de 1993 y 1997 la volatilidad alcanza al 5,3\%. Luego, entre las votaciones de 1997 y 2001 esta experimenta un alza, asumiendo un valor de $8,0 \%$, para luego volver a descender entre 2001 y 2005 a un 5,5\%. La mayor volatilidad del período se registra entre el último par de elecciones (2005-2009), donde la volatilidad alcanza un valor de 10,2\%.

16 Para arribar a los resultados esperados a partir de la utilización de encuestas con preguntas retrospectivas es preciso sortear una serie de problemáticas propias de esta estrategia de análisis. La principal deriva de la existencia de ocultamiento en la intención de voto en la elección 2, o de la no recordación del comportamiento de voto en la elección anterior. Ambos problemas pueden presentarse de forma independiente o conjunta, invalidando en cualquiera de estos casos la comparabilidad de la información sobre la que se pretende contrastar el comportamiento de voto.

17 En vista de aquello, el análisis de los datos opera bajo el supuesto de que las submuestras de casos sin problemas de respuesta mantienen en lo sustancial la representatividad del Universo de votantes una vez ponderados los datos.

18 Pese a esto último, conviene no desestimar las limitantes asociadas a la estrategia de estudio utilizada. Trabajar con submuestras de casos sin problemas de respuesta puede introducir sesgos a las estimaciones, independientemente de que los resultados sean ponderados. En este caso la ponderación, si bien ajusta los resultados reales de la elección 2 de acuerdo con los resultados reales de dicha votación, distorsiona los de la elección 1 (a ello contribuye también la ausencia en la muestra de los casos representativos de aquellos segmentos de votantes que salen del padrón). 
TABLA 2

Volatilidad Interbloques 1993-2009

\begin{tabular}{|c|c|c|c|c|}
\hline PERÍODO $\rightarrow$ & $1993-1997$ & $1999-2001$ & $2001-2005$ & $2005-2009$ \\
\hline Volatilidad (interbloques) & $5,3 \%$ & $8,0 \%$ & $5,5 \%$ & $10,2 \%$ \\
\hline
\end{tabular}

Fuente: Elaboración propia a partir de los datos del Servicio Electoral

De acuerdo con estas cifras, la primera gran alteración se produjo entre las elecciones de 1997 y 2001 y luego entre el 2005 y el 2009. ¿Qué factores explican dicho aumento? Se podrían identificar una serie de factores, como el carácter más desafiante adoptado por la derecha (Avendaño, 2011), que le permite penetrar en nuevos segmentos del electorado, así como la escisión de sectores otrora parte del bloque Concertación, incrementando de esta forma la importancia electoral del sector "Otros" en la elección de 2009. Lo cierto es que, a pesar de este crecimiento, la volatilidad sigue presentándose de una forma más bien moderada.

La lectura tradicional de estos valores tendería a asumir que este marco de estabilidad se produce debido a que la mayor parte de los electores vota por la misma opción política entre una elección y otra. En términos operativos, de ser esto efectivo, los votantes tipo I predominarían en la composición del electorado en las diferentes elecciones contenidas en el período. Veamos, pues, qué ocurre a nivel de los electores.

El análisis de los datos de las Encuestas CERC indica que el porcentaje de votantes que mantienen un comportamiento de voto estable (votantes tipo I) no supera el 60,2\% en el conjunto de elecciones analizadas. Para la elección de 1997 el porcentaje de electores estables alcanza el 58,2\% y para la de 2001 el 60,2\%. En la votación de 2005 los votantes tipo I alcanzan el 53,1\% del total del electorado, y luego al 54,3\% en la última elección de 2009. Como vemos, el porcentaje de votantes estables dista considerablemente de alcanzar el complemento de $100 \%$ del nivel de volatilidad electoral interbloques observado para cada par de elecciones. Ello obliga a descartar la hipótesis de la estabilidad del votante como causa de la estabilidad de los resultados electorales. ¿Cómo se originan entonces estos bajos índices de volatilidad electoral?

Para intentar responder esta última pregunta debemos observar la distribución del cruce del comportamiento de voto declarado para cada par de elecciones. Veamos en primer lugar qué sucede con el electorado en 1997.

La información indica que el año 1997 el bloque Concertación obtuvo las preferencias de un $36,3 \%$ del total del electorado (correspondiente al 50,5\% del total de votos válidamente emitidos $)^{19}$. El $32,5 \%$ del total de votantes de dicho año era un electorado fiel a la Concertación,

19 Obtendríamos este porcentaje si excluyéramos del conteo a los casos que no emitieron un voto válido ("Ninguno"). 
TABLA 3

Comparación del comportamiento electoral entre elecciones de 1997 y 1993

\begin{tabular}{|l|c|c|c|c|c|c|c|}
\hline $\begin{array}{c}\text { BLOQUES } \\
1997 \\
\downarrow\end{array}$ & \multicolumn{7}{|c|}{ BLOQUES 1993 } \\
\cline { 2 - 8 } & CONCERT. & ALIANZA & IZQUIERDA & OTROS & NINGUNO & $\begin{array}{c}\text { NO } \\
\text { INSCRITO }\end{array}$ & TOTAL \\
\hline $\begin{array}{l}\text { Concertación } \\
\text { Alianza }\end{array}$ & 32,5 & 0,6 & 0,4 & 0,4 & 1,2 & 1,2 & 36,3 \\
\hline Izquierda & 1,5 & 0,0 & 4,7 & 0,0 & 0,5 & 0,7 & 7,5 \\
\hline Otros & 0,4 & 0,2 & 0,1 & 1,0 & 0,3 & 0,1 & 2,0 \\
\hline Ninguno & 10,3 & 0,9 & 0,4 & 0,9 & 14,3 & 1,3 & 28,2 \\
\hline Total & 47,0 & 21,7 & 5,8 & 2,7 & 17,6 & 5,2 & 100 \\
\hline
\end{tabular}

Fuente: Elaboración propia a partir de los datos del Servicio Electoral y la Encuesta CERC.

pues proyectaba votar por este bloque en aquella elección, habiéndolo hecho también en 1993. Dicho porcentaje alcanzó el 20\% en el caso del bloque Alianza (actual Coalición por el Cambio), el 4,7\% para la Izquierda y el 1\% para Otras opciones políticas. La suma de estos porcentajes origina el 58,2\% de votantes estables para la elección de 1997. En tanto, un 6,5\% del electorado manifiesta un comportamiento móvil (votante tipo II), pues proyecta votar en la elección de 1997 por candidatos de un conglomerado diferente al escogido en la votación precedente. Dentro de este porcentaje observamos que un 2,3\% del electorado cambió su preferencia de votación de la Concertación en 1993 a la Alianza en 1997, mientras que solo un 0,6\% realizó el cambio inverso (nótese que ningún elector cambia su preferencia de la Alianza en 1993 a la Izquierda en 1997). Por su parte, el electorado tipo III alcanza al 19,7\% del total de votantes en 1997. Como se señaló con anterioridad, el votante tipo III es aquel que vota en solo una de las dos elecciones, pues en la otra o bien no emite un voto por opción política (nulo, blanco, no concurrente) o no formaba parte del padrón en la elección de 1993. Dentro de este porcentaje, la tendencia más destacada viene dada por el 10,3\% del electorado que votó por la Concertación en 1993 y que proyecta no emitir un voto válido en la elección de 1997. En total, los votantes tipo III que podrían ser catalogados como "desencantados pasivos" (votan en 1993 y no lo hacen en 1997) ascienden al 12,5\% del electorado en 1997. En tanto, aquellos que recorren el camino inverso (no votan en 1993 pero sí lo hacen en 1997), a los cuales podríamos llamar "reencantados" 20 , alcanzan el 7,2\% del total de electores. Por su parte, un $14,3 \%$ del electorado se mantendría desvinculado de los procesos electorales, al

20 La denominación resulta útil, pero es inexacta, pues forman parte de este segmento votantes no inscritos en la elección de 1993. 
no votar por ninguna opción política en ambas elecciones (votante tipo IV). Finalmente, el electorado tipo $\mathrm{V}$ asciende al 1,3\% del total de inscritos en 1997.

De acuerdo con lo anterior, observamos una importante presencia de votantes no estables en la composición del electorado. En conjunto, los votantes tipo II y III agrupan al 26,2\% del total padrón electoral en 1997. Ambos tipos de electores tienen en común el que su situación para efectos del recuento electoral es diferente en ambos comicios, razón por la cual su presencia podría generar cambios en las votaciones de las diferentes coaliciones políticas, a menos de que su comportamiento presente en alguna medida un carácter autocompensado. Esto último es precisamente lo que se observa en los datos. Todos los conglomerados pierden y ganan votantes, razón por la cual los resultados de votación varían efectivamente, pero lo hacen en términos moderados.

Pese a las limitantes del procedimiento para efectos de "cuadrar" las variaciones de votación estimadas con los resultados electorales reales (lo cual deriva de la estrategia metodológica utilizada), el análisis de los datos obtenidos permite extraer conclusiones acerca del sustento de la estabilidad electoral a partir de la composición del electorado en términos de la tipología de votantes adoptada. Vistos a esta escala, los resultados indican que el cambio moderado en los porcentajes de votación entre 1993 y 1997 (volatilidad 5,3\%) no responde al predominio de votantes estables en la composición del electorado, sino más bien, a la existencia de transferencias de votación de signo inverso (movilidad), y a variaciones en la composición del electorado efectivo en 1997 en comparación a lo observado en la elección de 1993.

Una tendencia similar se observa en el resto de las elecciones analizadas. En todas aquellas los conglomerados ganan y pierden electores en comparación con las votaciones precedentes. De allí que los rangos de estabilidad electoral observados deban ser atribuidos nuevamente al carácter autocompensado de los cambios asociados a la presencia de votantes no estables. Las siguientes tres tablas presentan el cruce en el comportamiento de votación declarado para los intervalos 1997-2001, 2001-2005 y 2005-200921.

\subsection{Síntesis de resultados}

Los datos muestran un leve decrecimiento del porcentaje de votantes que mantienen un comportamiento de voto estable, desde un 58,2\% en 1997 hasta un 54,3\% en 2009, versus un incremento del conjunto de electores no estables, los cuales pasaron de ser el $26,2 \%$ en 1997 al 36,9\% en la elección parlamentaria de 2009. Los votantes desvinculados oscilan entre un $14,3 \%$ en la primera elección de la serie analizada, hasta un 5,3\% en 2009. Por su parte, los electores tipo $V$ se incrementan desde el 1,3\% en 1997 hasta ser un 6,9\% en la última elección parlamentaria. Probablemente se trata de un perfil de votantes que se inscribieron en los registros electorales con interés en participar solo en las elecciones presidenciales (coincidentes con las de cámara baja en los años 2005 y 2009), razón por la cual no emiten un voto válido en las elecciones de cámara baja aquí analizadas. De allí,

\footnotetext{
21 Para una descripción más detallada de estos resultados, ver Avendaño y Sandoval (2012).
} 
TABLA 4

Comparación del comportamiento electoral entre elecciones de 2001 y 1997

\begin{tabular}{|l|c|c|c|c|c|c|c|}
\hline \multirow{2}{*}{$\begin{array}{c}\text { BLOQUES } \\
2001 \\
\downarrow\end{array}$} & \multicolumn{7}{|c|}{ BLOQUES 1997 } \\
\cline { 2 - 8 } & CONCERT. & ALIANZA & IZQUIERDA & OTROS & NINGUNO & $\begin{array}{c}\text { NO } \\
\text { INSCRITO }\end{array}$ & TOTAL \\
\hline Concertación & 32,5 & 0,5 & 0,6 & 0,2 & 1,4 & 2,0 & 37,2 \\
\hline Alianza & 3,6 & 26,4 & 0,2 & 0,3 & 2,9 & 0,7 & 34,0 \\
\hline Izquierda & 2,0 & 0,0 & 1,8 & 0,0 & 0,8 & 0,3 & 4,8 \\
\hline Otros & 0,3 & 0,4 & 0,0 & 0,3 & 0,0 & 0,1 & 1,2 \\
\hline Ninguno & 7,8 & 1,8 & 0,3 & 0,5 & 12,2 & 0,3 & 22,8 \\
\hline Total & 46,1 & 29,1 & 2,8 & 1,4 & 17,2 & 3,3 & 100 \\
\hline
\end{tabular}

Fuente: Elaboración propia a partir de los datos del Servicio Electoral y la Encuesta CERC.

TABLA 5

Comparación del comportamiento electoral entre elecciones de 2005 y 2001

\begin{tabular}{|l|c|c|c|c|c|c|c|}
\hline \multirow{2}{*}{$\begin{array}{c}\text { BLOQUES } \\
2005 \\
\downarrow\end{array}$} & \multicolumn{7}{|c|}{ BLOQUES 2001 } \\
\cline { 2 - 8 } & CONCERT. & ALIANZA & IZQUIERDA & OTROS & NINGUNO & $\begin{array}{c}\text { NO } \\
\text { INSCRITO }\end{array}$ & TOTAL \\
\hline Concertación & 32,8 & 0,6 & 0,2 & 0,3 & 5,9 & 2,0 & 41,9 \\
\hline Alianza & 3,8 & 19,0 & 0,1 & 1,6 & 4,4 & 2,3 & 31,2 \\
\hline Izquierda & 2,0 & 0,0 & 1,3 & 0,2 & 1,6 & 0,9 & 6,0 \\
\hline Otros & 0,0 & 1,1 & 0,0 & 0,0 & 0,0 & 0,0 & 1,1 \\
\hline Ninguno & 5,9 & 2,4 & 0,2 & 5,2 & 4,7 & 1,4 & 19,8 \\
\hline Total & 44,5 & 23,0 & 1,9 & 7,3 & 16,7 & 6,6 & 100 \\
\hline
\end{tabular}

Fuente: Elaboración propia a partir de los datos del Servicio Electoral y la Encuesta CERC. 
TABLA 6

Comparación del comportamiento electoral entre elecciones de 2009 y 2005

\begin{tabular}{|l|c|c|c|c|c|c|c|}
\hline \multirow{2}{*}{$\begin{array}{c}\text { BLOQUES } \\
2009 \\
\downarrow\end{array}$} & \multicolumn{7}{|c|}{ BLOQUES 2005 } \\
\cline { 2 - 8 } & CONCERT. & ALIANZA & IZQUIERDA & OTROS & NINGUNO & $\begin{array}{c}\text { NO } \\
\text { INSCRITO }\end{array}$ & TOTAL \\
\hline Concertación & 26,9 & 0,5 & 0,2 & 0,1 & 1,3 & 4,8 & 33,9 \\
\hline Alianza & 2,9 & 25,5 & 0,0 & 0,0 & 1,9 & 4,2 & 34,5 \\
\hline Izquierda & 1,2 & 0,0 & 1,9 & 0,0 & 0,1 & 1,8 & 5,1 \\
\hline Otros & 2,4 & 0,8 & 0,2 & 0,0 & 0,9 & 1,7 & 6,0 \\
\hline Ninguno & 5,7 & 1,8 & 0,6 & 0,2 & 5,3 & 6,9 & 20,6 \\
\hline Total & 39,1 & 28,6 & 2,9 & 0,3 & 9,6 & 19,5 & 100 \\
\hline
\end{tabular}

Fuente: Elaboración propia a partir de los datos del Servicio Electoral y la Encuesta CERC.

hemos decidido finalmente denominar a este perfil de electores como "votantes alternos". La siguiente tabla sintetiza la composición del electorado por tipo de votantes para las elecciones realizadas entre 1997 y 2009.

TABLA 7

Composición del electorado según tipos de votantes 1997-2009

\begin{tabular}{|l|c|c|c|c|}
\hline & 1997 & 2001 & 2005 & 2009 \\
\cline { 2 - 5 } & $\%$ & $\%$ & $\%$ & $\%$ \\
\hline Tipo I (votante estable) & 58,2 & 60,2 & 53,1 & 54,3 \\
\hline Tipo II (votante móvil) & 6,5 & 8,1 & 9,9 & 8,8 \\
\hline Tipo III (desencantado/reencantado) & 19,7 & 18,6 & 30,8 & 28,1 \\
\hline Tipo IV (desvinculado) & 14,3 & 12,2 & 4,7 & 5,3 \\
\hline Tipo V (votante alterno) & 1,3 & 0,3 & 1,5 & 6,9 \\
\hline Total & 100 & 100 & 100 & 100 \\
\hline
\end{tabular}

Fuente: Elaboración propia a partir de datos encuestas CERC. 
De acuerdo con estos datos, el incremento en el peso relativo de votantes tipo III y el decrecimiento de electores desvinculados constituyen las tendencias más destacadas en los datos. Ello indica que los cambios de composición del electorado efectivo se han vuelto relevantes en el transcurso del período, tendencia marcada por el aumento del porcentaje de votantes "reencantados" en comparación con un decrecimiento de desencantados pasivos y de votantes desvinculados. El gráfico a continuación muestra la composición del votante tipo III en las votaciones analizadas.

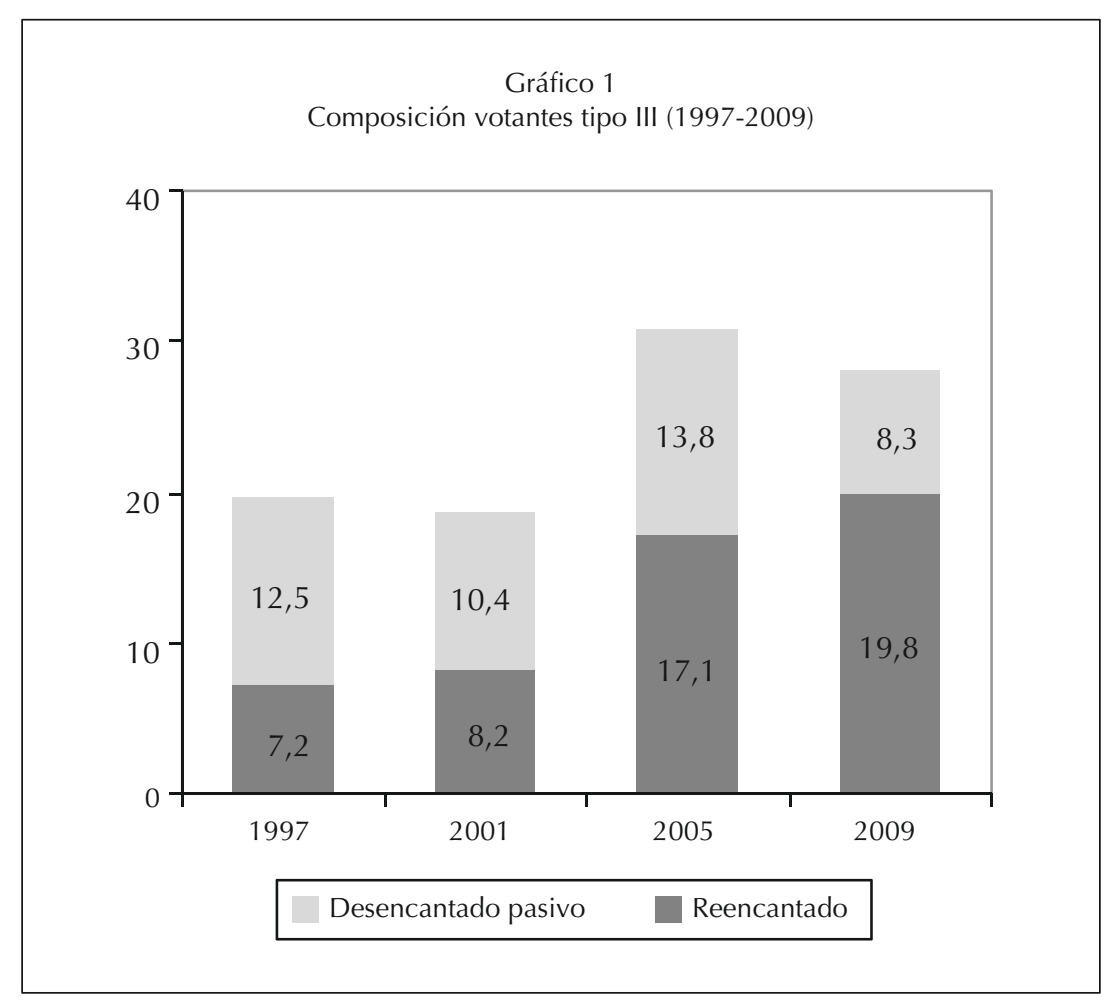

Contrastando estos resultados con las hipótesis iniciales del estudio, podemos concluir que:

- La volatilidad interbloques mantiene efectivamente niveles bajos o moderados en el conjunto de votaciones analizadas.

- El porcentaje de votantes estables en la composición del electorado ha disminuido en el transcurso del período.

- Entre cada par de elecciones estudiadas, todos los bloques ganan y pierden votación, lo que permite concluir que el voto no estable presenta un carácter autocompensado. 


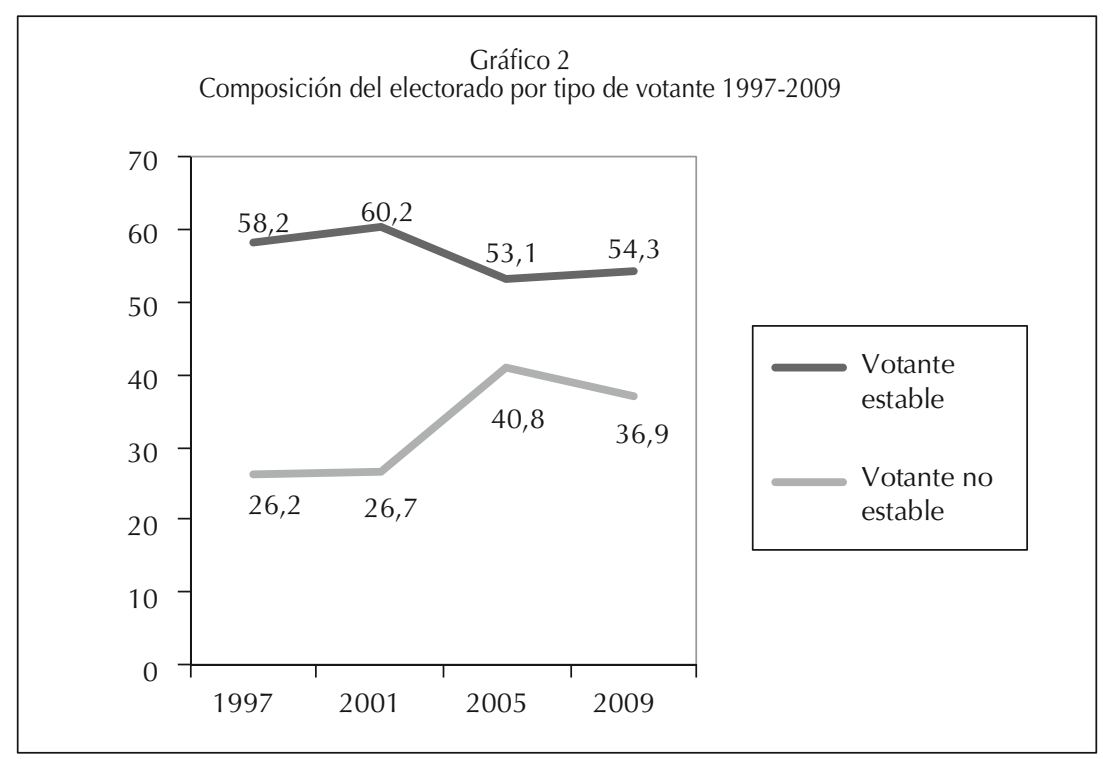

\section{CONCLUSIONES}

La estabilidad atribuida al sistema de partidos chileno es consecuencia de un diagnóstico parcial que ha puesto énfasis, principalmente, en la permanencia de los resultados electorales obtenidos por las dos coaliciones o bloques políticos predominantes desde 1989. Al mismo tiempo, se ha incurrido muchas veces en una verdadera "falacia ecológica" al suponer que la estabilidad reflejada a nivel de los resultados estaría relacionada con un comportamiento estable en el electorado. En otras palabras, de acuerdo con este supuesto, los electores no tenderían a modificar sus decisiones y adhesiones, sino más bien a mantener sus preferencias a lo largo del tiempo. Por el contrario, los datos aquí presentados muestran que la presencia de votantes estables es considerablemente menor a lo supuesto por los marcos interpretativos imperantes, e inferior incluso a lo reportado en estudios previos sobre el incremento del voto no estable (Huneeus, 2002; Inap-Segpres, 2008; López, 2008).

Lo anterior tiene una serie de implicancias para efectos del análisis de las problemáticas planteadas en este trabajo. En primer lugar, como hemos expuesto antes, el incremento del voto no estable, a la luz de la mantención de niveles de volatilidad bajos o moderados, indica que los rangos de estabilidad electoral observados se producen necesariamente como el resultado neto del carácter autocompensado de este conjunto de cambios. Los datos indican que todos los sectores políticos logran captar votantes, al tiempo que pierden un segmento de votantes otrora de estos sufragantes. Luego, el saldo positivo o negativo en este conjunto de cambios es lo que genera las variaciones de votación moderadas experimentadas por todos estos conglomerados. De allí se originan los bajos índices de volatilidad que muestra el sistema de partidos chileno.

En segundo término, los datos obtenidos indican que la presencia de votantes estables ha disminuido efectivamente durante el período, pero que dicha disminución ha sido menos 
drástica de lo esperado, esto último porque en realidad este perfil de votantes nunca superó el $60 \%$ del total del electorado en el conjunto de elecciones analizadas. Vale decir, en términos de la composición del electorado, nuestros datos no muestran un descenso abrupto de la presencia de votantes estables en torno a determinada data, como señalaron Joignant y López (2005), sino más bien que estos han mantenido un porcentaje, si bien mayoritario, no predominante a lo largo del período. Por su parte, la presencia de votantes móviles se incrementó en un rango similar a la disminución del voto estable. En la última elección de 2009 , cerca del $10 \%$ del electorado, es decir, del total de personas en ese entonces inscritas, votó por una opción política diferente a la escogida en 2005.

Ambas tendencias dan cuenta de una disminución de la incidencia de la estructura de clivajes en la determinación del comportamiento electoral en Chile. No obstante, el que sobre el $50 \%$ del electorado continúe votando de forma estable indica que dicha estructura, y particularmente el contenido del clivaje político, ha seguido manteniendo vigencia para buena parte del electorado. Para los votantes móviles, en cambio, dicho límite políticoideológico parece haber perdido relevancia. Por su parte, los votantes desencantados pasivos y desvinculados constituyen un segmento para el cual no solo el contenido del clivaje, sino la dinámica misma del sistema democrático, parece perder importancia. Sin embargo, no se trata de una desvinculación definitiva, tal como demuestra el incremento en la presencia de votantes "reencantados" en la composición del electorado. Esta última constituye una de las tendencias más destacadas de los resultados, e indica que durante las últimas elecciones votantes que no habían votado en elecciones precedentes han vuelto a sufragar, o se han inscrito para hacerlo. De este modo, los índices de desafección parecen no transformarse en un alejamiento definitivo con el sistema democrático, sino más bien, variar en función del carácter más o menos convocante de una elección determinada.

El escenario parece estar marcado entonces por una constante rotación del electorado. Electores que habían dejado de votar, vuelven a hacerlo en comicios que consideran más relevantes; asimismo lo hacen votantes que no formaban antes del padrón. Otros que habían votado, dejan de hacerlo, probablemente declarándose desencantados con el sistema. El resultado neto de este conjunto de cambios son los índices de volatilidad bajos o moderados observados en el sistema chileno, siendo esta última una característica presente a lo largo de todo el período analizado. La diferencia entre las elecciones de 1997 y 2001 respecto de las dos últimas, consiste en el incremento de los votantes "reencantados" y el decrecimiento de desvinculados y desencantados pasivos.

En el plano de las vinculaciones entre sistema de representación y sociedad, el escenario calza con la descripción realizada por Luna (2008) en torno al advenimiento de una "nueva configuración" definida por la convivencia de vínculos programáticos y no programáticos, colectivos y no colectivos, antes que la primacía de un vínculo políticamente estructurado. El concepto de "voto no estable autocompensado" permite armonizar la descripción de Luna con la mantención de la estabilidad electoral a nivel agregado. El comportamiento de voto no estable (votantes tipo II y III), junto con la desvinculación (tipo IV), constituyen la expresión del incremento de la desafección 
política a nivel electoral, mientras que la permanencia de votantes estables permite representar la vigencia del vínculo político-programático. Visto así, el escenario presente hasta 2009 se muestra dividido en dos mitades.

Lo analizado en este artículo remite a un período en el que estuvo vigente el sistema de inscripción voluntaria y de voto obligatorio. No obstante, el comportamiento asumido por el electorado en este período sirve de antecedente para entender las dinámicas que se puedan ir dando desde la puesta en marcha del voto voluntario, como ocurrió a partir de las elecciones municipales de octubre de 2012. De acuerdo con el comportamiento que han ido manifestando los votantes hasta las elecciones de 2009, el voto voluntario podría tender a acrecentar el problema de representatividad y de distanciamiento con los principales bloques políticos. Una de las consecuencias, en un período significativo de tiempo, será la alteración en el porcentaje de votantes fijos, cuestión ya evidenciada en los comicios municipales de 2012, y desde luego en la composición de ese tipo de votantes. Asimismo, podrá afectar la participación entre cada elección, incrementando el número de electores tipo III y, sobre todo, el porcentaje de no concurrentes. Son diversos los estudios y las evidencias internacionales que dan cuenta de un comportamiento diferenciado entre los electores, en países que cuentan con voto voluntario. Tal diferenciación se reconoce principalmente en una menor participación e identificación partidaria, que se da especialmente entre la población débilmente integrada y con bajos niveles de escolaridad. Si bien el presente estudio no indaga en los factores socioeconómicos que inciden en el comportamiento del electorado, deja entrever un cuadro diferenciado entre los electores que se tenderá a acrecentar con la carencia de un estímulo objetivo para la participación.

Por último, cabe agregar que si bien el análisis realizado remite a un período en el que estuvo vigente otro sistema de inscripción y participación electoral (inscripción voluntaria y voto obligatorio), el comportamiento asumido por el electorado en dicho período puede servir de antecedente para entender las dinámicas que se puedan ir dando desde la puesta en marcha del voto voluntario, como ocurrió a partir de las elecciones municipales de octubre de 2012. De acuerdo con los datos mencionados, y al comportamiento de los votantes hasta las elecciones de 2009, el sufragio voluntario podría tender a acrecentar el problema de representatividad y de distanciamiento con los principales bloques políticos. Una de las consecuencias, en un período significativo de tiempo, será sin duda la alteración en el porcentaje de votantes estables en la composición del electorado, cuestión ya evidenciada en los comicios municipales de 2012. Asimismo, podrá afectar la participación entre cada elección, incrementando el número de electores tipo III y, sobre todo, el porcentaje de votantes desvinculados.

\section{BIBLIOGRAFÍA}

Alcántara, Manuel (2004): "Partidos políticos en América Latina: precisiones conceptuales, estado actual y retos futuros", Revista Estudios Políticos, 124, pp. 54-95.

Angell, Alan (2003): "Party change in Chile in comparative perspective", Revista de Ciencia Política, 23 (2), pp. 49-66. 
Avendaño, Octavio (2011): “La oposición política en Chile durante el período 1990-2011. Una aproximación conceptual y empírica", Revista de Ciencias Sociales, 8, pp. 147-186.

Avendaño, Octavio y Pablo Sandoval (2011): "Movilidad y volatilidad. Una propuesta de medición de la incidencia de los factores de inestabilidad electoral", Universidad de Chile, Departamento de Sociología, Santiago, en http://www.facso.uchile. cl/? nfpb=true\& pageLabel=conUrlFacso\&url=75435

(2012): “Estabilidad electoral y desafección política en Chile 1993-2009. Análisis acerca de la volatilidad electoral y el voto no estable", en Daniel Bello (ed.): Elecciones, participación política y calidad de la democracia en América Latina, LOM Ediciones, Santiago, pp. 145-174.

Bartolini, Stefano y Peter Mair (1990): Identity, competition, and electoral availability. The stabilization of European electorates 1885-1985, Cambridge University Press, Cambridge.

Crewe, Ivor y David Denver (1985): Electoral change in western democracies, Croom Helm, London.

Gamboa, Ricardo (2006): "El establecimiento del sistema binominal”, en Carlos Huneeus (comp.): La reforma al sistema binominal en Chile. Propuestas para el debate, Catalonia, Santiago, pp. 45-74.

Garretón, Manuel Antonio (2000): La sociedad en que vivi(re)mos: introducción sociológica al cambio de siglo, LOM Ediciones, Santiago.

Gutiérrez, Alexis y Miguel Ángel López (2007): “Factores explicativos de la conducta electoral de los chilenos", en C. Huneeus, F. Berríos y R. Gamboa (eds.): Las elecciones chilenas de 2005: partidos, coaliciones y votantes en transición, Catalonia, Santiago, pp. 177-195.

Huneeus, Carlos (2002): “¿Dónde se fueron los votantes del PDC?”, Asuntos Públicos, Informe n¹75. (2003): Chile, un país dividido. La actualidad del pasado, Catalonia. Santiago.

Instituto de Asuntos Públicos y Secretaría General de la Presidencia (INAP-SEGPRES) (2008): La estabilidad del voto y su fidelidad histórica en Chile desde la perspectiva de las matrices culturales históricas, Secretaría General de la Presidencia (SEGPRES), Santiago.

Joignant, Alfredo (2010): "Political parties in Chile: stable coalitions, inert democracy", en K. Lawson y J. Lanzaro (eds.): Political parties and democracy. Vol. 1: The Americas, Praeger, Wesport, pp. 127-147.

Joignant, Alfredo y Miguel Ángel López (2005): "Le comportement électoral au Chili: paradoxes et présomptions sur la continuité ou la rupture de l'orientation du vote", Problèmes d'Amérique Latine, 56, pp. 63-80.

Jones, Mark (2005): The role of parties and party systems in the policymaking process, trabajo presentado en "State reform, public policies and policy-making process", Banco Interamericano de Desarrollo, Washington D.C., febrero-marzo.

Lipset, Seymour M. y Stein Rokkan (1967): “Cleavage structures, party systems, and voter alignments: an Introduction", en S. M. Lipset y S. Rokkan (eds.): Party systems and voter alignments, The Free Press, Nueva York, pp. 1-64.

López, Miguel Ángel (2004): “Conducta electoral y estratos económicos: el voto de los sectores populares en Chile", Política, 43, pp. 285-298.

(2008): Conducta electoral en Chile: la paradoja de la estabilidad, paper preparado para ser presentado en el "IV Congreso Latinoamericano de Ciencia Política-ALACIP", San José de Costa Rica, 5-7 agosto. 
López, Miguel Ángel y Jaime Baeza (2011): "Las elecciones chilenas de 2009-10: ¿se derechizó el país?", en Manuel Alcántara Sáez y María Laura Tagina (eds.): América Latina: política y elecciones del bicentenario (2009-2010), Centro de Estudios Políticos y Constitucionales, Madrid, pp. 277-302.

Luna, Juan Pablo (2008): "Partidos políticos y sociedad en Chile. Trayectoria histórica y mutaciones recientes", en A. Fontaine, C. Larroulet, J. Navarrete e I. Walker (eds): Reforma de los partidos políticos en Chile, CEP-PNUD, Santiago, pp. 75-124.

Luna, Juan Pablo y David Altman (2011): “Uprooted but stable. Chilean party system and the concept of party system institutionalization", Latin America Politics and Society, 2 (56), pp. 1-28.

Mainwaring, Scott y Edurne Zoco (2007): "Political sequences and stabilization of interparty competition: electoral volatility in old and new democracies", Party politics, 13 (152), pp. 155-178.

Mainwaring, Scott y Timothy Scully (eds.) (1996): La construcción de instituciones democráticas: Sistemas de partidos en América Latina, CIEPLAN, Santiago.

Mainwaring, Scott y Mariano Torcal (2005): "La institucionalización de los sistemas de partidos y la teoría del sistema partidista después de la tercera ola democratizadora", América Latina Hoy, Universidad de Salamanca, 41, pp. 141-173.

Martínez, Javier y Margarita Palacios (1991): "El voto cambiante y la distancia social a la política", Proposiciones, 20, pp. 34-58.

Montes, Juan Esteban, Eugenio Ortega y Scott Mainwaring (2000): "Rethinking the Chilean Party System", Journal of Latin American Studies, 32 (3), pp. 795-824.

Morales, Mauricio (2010): Disolución de la identificación partidaria en Chile. Encuesta Nacional UDP 2010, Ediciones Universidad Diego Portales, Santiago, pp. 45-59.

Ortega F., Eugenio (2003): “Los partidos políticos chilenos: cambio y estabilidad en el comportamiento electoral 1990-2000", Revista de Ciencia Política, XXIII (2), pp. 109-147.

Pedersen, Mogens N. (1979): "The dynamics of West European party systems: Changing patterns of electoral volatility", European Journal of Political Research, 7, pp. 1-26.

PNUD (2002): Desarrollo Humano 2002. Nosotros los chilenos: un desafío cultural, PNUD, Santiago.

Powell, Eleanor y Tucker, Joshua (2009): “New approaches to electoral volatility: Evidence from Postcommunists countries", APSA 2009 Toronto Meeting Paper.

Roblizo, Manuel J. (2001): Transición a la democracia y evolución del comportamiento electoral en Bulgaria 1989-1994, Ediciones Universidad de Castilla-La Mancha, Cuenca, 2001.

Scully, Timothy (1996): "La reconstitución de la política de partidos en Chile", en S. Mainwaring y T. Scully: La construcción de instituciones democráticas: sistemas de partidos en América latina, CIEPLAN, Santiago, pp. 83-112.

Shair-Rossenfield, Sarah (2008): Assessing the causes and effects of electoral volatility: party system fragmentation, time and executive turnover, Thesis (MA)-The University of North California, Chapel Hill.

Siavelis, Peter (1999): "Continuidad y transformación del sistema de partidos en una transición 'modelo'", en P. Drake e I. Jaksic (comp.): El modelo chileno. Democracia y desarrollo en los noventa, LOM Ediciones, Santiago, pp. 223-256.

Tironi, Eugenio, Felipe Agüero y Eduardo Valenzuela (2001): "Clivajes políticos en Chile: perfil de los electores de Lagos y Lavín", Perspectivas, 5 (1), pp. 73-87. 
Valenzuela, Samuel (1995): "Orígenes y transformaciones del sistema de partidos en Chile", Estudios Públicos, 58, pp. 5-80.

(1999): "Reflexiones sobre el presente y futuro del paisaje político chileno a la luz del pasado", Estudios Públicos, 75, pp. 273-290.

Recibido: 25-06-2013

Aceptado: 30-07-2013 